\title{
The SKA as a Direct-to-Earth Data Acquisition Facility for Deep Space Science Missions
}

\author{
P.A. Fridman, L.I. Gurvits, S.V. Pogrebenko
}

\author{
JIVE, Oudehoogeveensedijk 4, Dwingeloo, 7991PD, The Netherlands
}

\begin{abstract}
The Square Kilometre Array is conceived as a next generation radio astronomy facility superior to any existing telescope primarily in sensitivity terms. The science drivers of the SKA project do not include data acquisition from and tracking of space missions. However, as a uniquely sensitive receiving radio facility on the planet in the foreseeable future, the SKA might have an important role as an ad-hoc addition to the network of Earth-based assets of space science and exploration programmes. In this contribution we assess the potential of the SKA as a receiving facility for Direct-to-Earth (DtE) delivery of science data from deep space missions. This assessment assumes DtE support to a prospective outer planet mission, such as the joint ESA-NASA Europa Jupiter System Mission (EJSM). We show that as a piggy-back on nominal science operations with practically no special hard- or software task-specific additions, SKA can offer acquisition of data streams with the rate of tens bps through low-power omni-directional transmission from spacecraft at a distance up to $10 \mathrm{AU}$. Such the support, especially during short mission-critical operations, might be extremely valuable means of enhancing science return and efficiency of deep space expeditions.
\end{abstract}

\section{Introduction}

Radio astronomy and space exploration are intertwined throughout the entire history of space flights, for more than half a century. One might recall the detection of the upper stage of the rocket placed in orbit the first artificial satellite, Sputnik, by the venerable Lovell telescope at Jodrell Bank in the UK in October 1957 as an example of radio astronomy support to the exploration of Space. Twelve years later, in July 1969, it was a high day for the Parkes Radio Telescope in Australia which turned to be the only Earth-based facility able to receive the historical TV translation of the Apollo-11 crew making first steps on the Moon. The format of this contribution does not allow us to provide a comprehensive review of mutually beneficial interactions between radio astronomy and exploration of space. But without any doubts, these interactions will continue in the future, during the SKA era. The study presented here aims at assessing the potential of the SKA as a receiving facility for Direct-to-Earth delivery of science data from deep space missions. Usually, these missions are equipped with either high-gain antennas for broad-band transmission of science data to Earth or operate with data relay via orbiters or fly-by spacecraft. In both cases, the data rate of modern and prospective missions measured in tens of kilobits per second and higher. However, as a back-up to the nominal data delivery schemes and recovery operations during and after non-nominal behaviour of a spacecraft (e.g. in safe-mode), a receipt of data streams of merely bits per second are extremely valuable. As demonstrated in the Huygens mission on Titan in 2005, just a detection of the Probes carrier tone transmitted toward the orbiting Cassini spacecraft (eavesdropping, in effect) makes substantial impact on the overall outcome of the mission. This regime of eavesdropping of usually weak signal transmitted through a low-directivity antenna is extremely difficult if possible at all even for the most sensitive dedicated deep space communication facilities. The overwhelmingly superior sensitivity of the SKA will make it possible to eavesdrop on the weak ra dio signals from planetary probes with acceptable data rates. This kind of support is particularly valuable for short in time mission-critical operations and can be accommodated without any serious impact on SKA operations. The latter is particularly true in view of the planned multi-view multi-user capability of the SKA.

A broad variety of engineering considerations dictate use of lower frequencies for science data delivery from small planetary probes to larger and powerful orbiters. In the case of Cassini-Huygens, the radio link from the descending probe to the orbiter were conducted at the frequency of $2 \mathrm{GHz}$. UHF bands (400 MHz) are being considered for up-link from Ganymede and Europa micropenetrators to the orbiter spacecraft of the joint ESA-NASA Europa Jupiter System Mission (EJSM) design study. Such the up-link is to be conducted through a low-power transmitter and low-directivity transmitting antenna. Similarly, the international ExoMars mission which is expected to blast off toward Mars later in this decade will use the UHF radio communication equipment. All the above missions and many others will be in operational phase beginning from 2020 a perfect match to the SKA timetable.

Although the prospective planetary missions are designed to deliver the bulk of science and housekeeping data through the high-capacity Ka-band radio channels $(32 \mathrm{GHz})$, the lower frequency band, such as $\mathrm{X}-(8 \mathrm{GHz}), \mathrm{S}(2 \mathrm{GHz})$ and UHF (800 and $400 \mathrm{MHz}$ ) will remain active in the arsenal of deep space communications. These lower frequency operations are perfectly consistent with the operational range of the SKA.

Communication with deep space missions usually employs dedicated Earth-based facilities equipped with large antennas (such as the NASA's Deep Space Network, DSN). The most commonly used scheme is that of duplex communication. High gain antennas and powerful transmitters onboard spacecraft (S/C) allow communication to be maintained up to the edges of the Solar System. However, the radio link system imposes 
serious restrictions on the mission operations. The restrictions grow rapidly with the increase of the distance to the spacecraft. These restrictions are particularly severe for short-living planetary probes. In most cases, planetary probes relay science and housekeeping telemetry data via orbiters or flyby S/C. Usually, such the scheme requires buffering the probe's data onboard the relay $\mathrm{S} / \mathrm{C}$. The Cassini-Huygens mission is an example of such the communication scheme: the Huygens Probe communicated data from the descent phase of the mission to Titan via the Cassini spacecraft. As was demonstrated by the Huygens mission, an ad-hoc direct receipt of the Huygens carrier signal by Earth-based radio telescopes turned out to be a valuable asset and contributed to the overall success of the mission (Lebreton et al. 2005).

In the traditional relay scheme, a planetary probe is equipped with a low-gain antenna roughly oriented toward the relay S/C and not necessarily toward the Earth. The probe's transmitter power is limited to several watts (typically $<5 \mathrm{~W}$ ). Such a low power link makes it practically impossible to provide a down-link to Earth from the probe using any of the existing DSN-style facilities or presently operational radio telescopes (beyond detecting a carrier signal, and even this with considerable difficulties).

An alternative to the traditional relay scheme is a Direct-toEarth (DtE) communication method. It brings in a number of advantages, especially in the case of communicating data from planetary probes - landers, penetrators, atmosphere and surface vehicles, etc. DtE makes unnecessary an extremely costly component of a mission, a relay $\mathrm{S} / \mathrm{C}$. Even if the role of the relay $\mathrm{S} / \mathrm{C}$ is performed by an indispensable probe's carrier (e.g. as the Cassini $\mathrm{S} / \mathrm{C}$ in the Cassini-Huygens mission), the relay scheme imposes severe ballistic restrictions and other operational limitations, in particular, on timing and duration of the broadcast from the probe. All in all, the relay scheme is responsible for a very considerable fraction of the cost of one bit of scientific data delivered from the probe to Earth. Of course, the relay scheme enables considerably higher amount of data to be delivered from the probe to Earth than can be provided by DtE. But DtE can be considered as a backup to the relay scheme, especially during critical events of a probe's science programme.

Recently major space agencies began to assess design options for the next generation planetary probes. These missions are expected to reach their destinations and begin in-situ operations around 2020 or later. In Europe, under the ESA's Cosmic Vision programme, a number of proposals call for multi-spacecraft missions, with probes going to Titan (TandEM and TSSM study, Coustenis et al. 2008), Europa and Ganymede (Laplace and EJSM study, Blanc et al. 2008), Venus (EVE, Chassefière et al. 2008), ExoMars. Another class of missions include deep-diving probes into dense atmospheres of giant planets, e.g. the mission Kronos proposed under the ESA's Cosmic Vision programme (Marty et al. 2008). This mission aims to study the Saturn atmosphere in-situ at the pressure level of $\sim 10$ bar. Communication from such the probe is particularly difficult due to a considerable attenuation of the signal in dense atmosphere. Since attenuation grows with frequency, the probe broadcasts at frequencies below $1 \mathrm{GHz}$ - the range, at which radio telescopes are much more sensitive than standard DSN facilities. In all the cases above, the probes will broadcast at relatively low frequencies, certainly not higher than $8 \mathrm{GHz}$, making them suitable targets for radio telescopes operating at well established in radio astronomy practice $\mathrm{GHz}$ bands.

The huge effective area of the Square Kilometre Array (SKA) would enable DtE communication from a distant S/C located practically anywhere in the Solar System. The case for SKA as a S/C tracking facility is discussed by Jones (2004). Below we present estimates of SKA DtE options under various considerations for the probe's signal strength and modulation.

\section{Radio link power budget}

We analyse the SKA DtE communication scheme under the following assumptions:

1. The distance from Earth to a transmitting probe is $R=5.0-10.0 \mathrm{AU}$ :

2. The communication is conducted at UHF-, S- and X-bands on the standard frequencies of 400, 2.3 and $8.4 \mathrm{MHz}$, respectively.

3. The probe transmitter power is considered in the range $1-10$ $\mathrm{W}$.

$25 \%$ of this power is spent on the transmission of the unmodu- $\Omega$ lated carrier frequency signal, so in the following calculations $P_{\text {tr }}$ will be multiplied on 0.75 .

4. The probe's antenna is omnidirectional (i.e., the antenna gain $G_{t r}=1$ or $0 d B$ ).

5. The system equivalent flux densities (SEFD) are given in Table 1.

Table 1: System noise temperature and SEFD of three radio telescopes

\begin{tabular}{c|c|c|c|}
\hline & UHF-band & S - band & X-band \\
\hline \hline Name & SEFD, J & SEFD, J & SEFD, J \\
\hline Effelsberg & 84 & 20 & 20 \\
Arecibo & 11 & 3 & 6 \\
$\begin{array}{c}\text { SKA - } \\
\text { aperture array } \\
\text { SKA - }\end{array}$ & 1.6 & 0.92 & 1.84 \\
parabolic dishes & 2.15 & 1.23 & 1,84 \\
\hline
\end{tabular}

The two primary communication resources are the transmitted power and the channel bandwidth. In many communication systems, one of these resources is more precious than the other. Therefore, the communication systems are classified as either power-limited or bandwidth-limited. In power-limited systems, coding schemes are used to save power at the expense of bandwidth, whereas in band-limited systems spectrally efficient modulation techniques can be used to save bandwidth at the expense of power. We think that in deep space communication systems it is the available power which defines main design and operational restrictions. Hence, these systems may be classified as power-limited. 


\section{Bit error probability and transmission rate}

The signal-to-noise ratio (SNR) for the received signal in the bandwidth equal to $1 \mathrm{~Hz}$ is

$$
S N R_{\text {power }}=\frac{P_{t r} G_{t r} A_{\text {eff }}}{4 \pi R^{2} k T_{\text {sys }}}, k=1.23 \times 10^{-23} \mathrm{~J} / \mathrm{K} .
$$

The potential transmission bit rate can be estimated using the Shannon's formula for the capacity of a channel perturbed by additive white Gaussian noise with the bandwidth $\Delta F$ and a given channel $S N R_{\text {power }}$ as:

$$
C=\Delta F \log _{2}\left(1+\frac{S N R_{\text {power }}}{\Delta F}\right) .
$$

Fig. 1 shows the potential channel capacity for the distance 5.0 AU, $G_{t r}=1$ and $P_{\mathrm{tr}}=5 \cdot 0.75 \mathrm{~W}$ and the radio telescopes from Tables 1 .

This formula sets the limit on transmission rate but not on the error probability. In digital communication, another figure of merit rather than $S N R_{\text {power }}$ is used:

$$
S N R_{b i t}=S N R_{\text {power }} T_{b}=\frac{S N R_{\text {power }}}{R W},
$$

where $S N R_{b i t}$ is the signal-to-noise ratio per bit, $T_{b}$ is the duration of signal transmission per 1 bit and $R W$ is the transmission rate. Using this new notation and making the transmission bit rate equal to the channel capacity, $R W=C$, we get

$$
C=\Delta F \log _{2}\left(1+S N R_{b i t} \frac{C}{\Delta F}\right)
$$

and

$$
S N R_{b i t}=\frac{\Delta F}{C}\left(2^{C / \Delta F}-1\right)
$$

When $S N R_{b i t}$ decreases the ratio $(C / \Delta F) \rightarrow 0$ and the corresponding value $S N R_{b i t}=\frac{1}{\log _{2} e}=0.693$ or, in decibels $S N R_{b i t}=-1.6 \mathrm{~dB}$. This value of $S N R_{b i t}$ is called the Shannon limit.

In practice, it is not possible to reach the Shannon limit. For a bit error probability of $10^{-5}$, the binary phase-shift-keying modulation requires $S N R_{b i t}=9.6 \mathrm{~dB}$ (uncoded binary modulation). Therefore, for this case, the Shannon limit promises the existence of a theoretical improvement of $11.2 \mathrm{~dB}$ through the use of special coding (see section 4).

\subsection{BPSK and QPSK}

A digital modulation scheme with phase-shift keying (PSK) is widely used in space communication systems (Yuen et al. 1990). It provides both a low bit-error-rate (BER) and minimum bandwidth. For the case of the binary phase-shift keying (BPSK), BER can be estimated as follows:

$$
P_{\mathrm{BER}, \mathrm{BPSK}}=Q\left(\sqrt{2 \times S N R_{\mathrm{bit}}}\right)
$$

where

$$
Q(x)=\frac{1}{\sqrt{2 \pi}} \int_{x}^{\infty} e^{\frac{-t^{2}}{2}} d t,
$$

$S N R_{\text {bit }}=S N R_{\text {power }} \times T_{b i t}=\frac{S N R_{\text {power }}}{R W}$ is the signal-to-noise ratio per bit, $T_{b i t}$ is the duration of signal transmission per 1 bit and $R W=1 / T_{b i t}$ is the transmission rate.

Quadriphase shift keying ( $\mathrm{M}$-ary phase modulation, $\mathrm{M}=4$ ) , denoted by QPSK, has the same bit-error performance as BPSK, but the equivalent bit rate is two times higher than for BPSK .

Fig. 2 shows bit error probabilities as function of the transmission rate at $\mathrm{S}$-band calculated for several radio telescopes from Tables 1 , the distance $R=5.0 \mathrm{AU}, G_{t r}=1$ and BPSK.

In reality, the transmission antenna gain may significantly vary. Fig. 3 shows BER as a function of transmission rate for SKA and $G_{t r}$ as parameter.

The effective area of SKA as of the plane phase array is proportional to $\cos (\alpha)$ where $\alpha$ is the zenith angle of the target source. Fig. 4 shows BER as a function of the transmission rate for SKA and $\alpha$ as a parameter.

To obtain $B E R=10^{-5}$ for uncoded BPSK the nec-? essary $S N R_{b i t, B E R=10^{-5}}$ must be equal to $9.6 \mathrm{~dB}$ (or 9.099)! Therefore, the ratio $S N R_{\text {power }} / S N R_{b i t, B E R=10^{-5}}$ gives the transmission rate achievable for a given $S N R_{\text {power }}$ as a function of $P_{t r}, G_{t r}, R, A_{\text {eff }}$ for the value $B E R=10^{-5}$. Fig. 5 demonstrates how the transmission rate depends of the distance (in AU) for the radio telescopes in Tables, and $B E R=10^{-5}$, $P_{t r}=3.75 \mathrm{~W}, G_{t r}=1$.

Table 2 contains the values of transmission rate (bit/sec) for uncoded BPSK and the SKA core, $A_{\text {eff }}=10^{5} \cdot \cos (\alpha) \mathrm{m}^{2}$, $B E R=10^{-5}, T_{\text {sys }}=40 \mathrm{~K}$ (for aperture array, S-band) and $T_{\text {sys }}=60 \mathrm{~K}$ (for parabolic dishes, X-band), distance $=5 \mathrm{AU}$. The transmitter power $P_{t r}$, antenna gain $G_{t r}$ and $\alpha$ are variable parameters.

\subsection{MFSK}

Another option for increasing the transmission rate is based on the use of M-ary orthogonal signals. Every binary sequence of the length $k$ corresponds to one of the $M=2^{k}$ orthogonal signals which modulate carrier signals.

In the following example, the $\mathrm{M}$-ary frequency shift keying (MFSK) is used: each symbol corresponding to a particular binary sequence of the length $k$ is transmitted on one of the $m$-th frequencies during an interval $T$ :

$$
s_{m}(t)=A \cos \left[2 \pi\left(f_{c}+m \Delta f\right) t\right], \leq t \leq T, m=1,2, \ldots . M,
$$

where $\Delta f=1 / 2 T, T-$ is the duration of a symbol. For example, for $k=5$, there are 32 different orthogonal signals, each at its own frequency. The total bandwidth is $W=M \Delta f$. The effective transmission rate is $R W=k / T, k=\log _{2} M$. The ratio $R W / W$ is called bandwidth expansion: $R W / W=$ $\left(2 \log _{2} M\right) / M$. Theoretically, by increasing $M$ it is possible to reach the Shannon's limit.

The probability of bit error for the coherent processing of $M=2^{k}$ orthogonal signals can be calculated using the follow- 
Table 2: The DtE transmission rate (bit/sec) for uncoded BPSK and the SKA core, for S-band (aperture array) $A_{\text {eff }}=1.5 \cdot 10^{5} \cdot \cos (\alpha)$ $m^{2}, B E R=10^{-5}$, distance $=5 A U$, transmitter power $P_{t r} \cdot 0.75$ and antenna gain $G_{t r}, \eta=0.8, T_{s y s}=40 \mathrm{~K} ; \mathrm{X}$-band (parabolic dishes), $\eta=$ $0.6, T_{s y s}=60 \mathrm{~K}$; UHF-band (aperture array), $T_{s y s}=70 \mathrm{~K}$, additional $3-\mathrm{dB}$ loss in the Earth ionosphere, interplanetary medium and Jupiter atmosphere are taken into account.

UHF-band, aperture array

\begin{tabular}{|c|c|c|c|c|}
\hline \multicolumn{5}{|c|}{$\begin{array}{l}\text { UHF-band, aperture array } \\
\qquad \alpha=0^{\circ}\end{array}$} \\
\hline$P_{t r}, \mathrm{~W}$ & 1.0 & 3.0 & 5.0 & 10.0 \\
\hline$G_{t r}=0 d B$ & 0.73 & 2.19 & 3.64 & 7.28 \\
\hline$G_{t r}=5 d B$ & 2.30 & 6.91 & 11.51 & 23.03 \\
\hline$G_{t r}=10 d B$ & 7.28 & 21.85 & 36.41 & 72.82 \\
\hline \multicolumn{5}{|c|}{$\begin{array}{c}\text { S-band, aperture array } \\
\alpha=0^{\circ}\end{array}$} \\
\hline$P_{t r}, \mathrm{~W}$ & 1.0 & 3.0 & 5.0 & 10.0 \\
\hline$G_{t r}=0 d B$ & 2.55 & 7.65 & 12.74 & 25.49 \\
\hline$G_{t r}=5 d B$ & 8.06 & 24.18 & 40.30 & 80.59 \\
\hline$G_{t r}=10 \mathrm{~dB}$ & 25.49 & 76.46 & 127.43 & 254.86 \\
\hline \multicolumn{5}{|c|}{$\alpha=30^{\circ}$} \\
\hline$P_{t r}, \mathrm{~W}$ & 1.0 & 3.0 & 5.0 & 10.0 \\
\hline$G_{t r}=0 d B$ & 2.21 & 6.62 & 11.04 & 22.07 \\
\hline$G_{t r}=5 d B$ & 6.98 & 20.94 & 34.90 & 69.80 \\
\hline$G_{t r}=10 d B$ & 22.07 & 66.22 & 110.36 & 220.72 \\
\hline \multicolumn{5}{|c|}{$\alpha=60^{\circ}$} \\
\hline$P_{t r}, \mathrm{~W}$ & 1.0 & 3.0 & 5.0 & 10.0 \\
\hline$G_{t r}=0 d B$ & 1.27 & 3.82 & 6.37 & 12.74 \\
\hline$G_{t r}=5 d B$ & 4.03 & 12.09 & 20.15 & 40.30 \\
\hline$G_{t r}=10 \mathrm{~dB}$ & 12.74 & 38.23 & 63.72 & 127.43 \\
\hline \multicolumn{5}{|c|}{ X-band, parabolic dishes } \\
\hline$P_{t r}, \mathrm{~W}$ & 1.0 & 3.0 & 5.0 & 10.0 \\
\hline$G_{t r}=0 d B$ & 1.27 & 3.82 & 6.37 & 12.74 \\
\hline$G_{t r}=5 d B$ & 4.03 & 12.09 & 20.15 & 40.30 \\
\hline$G_{t r}=10 \mathrm{~dB}$ & 12.74 & 38.23 & 63.72 & 127.43 \\
\hline
\end{tabular}

ing expression (Proakis 2001, p. 259):

$$
\begin{aligned}
P_{\text {BER,FSK_C }}=\frac{2^{k-1}}{2^{k}-1} & \cdot \frac{1}{\sqrt{2 \pi}} \int_{-\infty}^{\infty}\left[1-\left(\frac{1}{\sqrt{2 \pi}} \int_{-\infty}^{y} e^{-\frac{t^{2}}{2}} d t\right)^{M-1}\right] \times \\
& \times \exp \left[-\left(y-\sqrt{2 \cdot S N R_{\text {symbol,FSK }}}\right)^{2} / 2\right] d y,(9)
\end{aligned}
$$

where $S N R_{\text {symbol,FSK }}=S N R_{\text {power }} \times T$, each signal transmits $k$ bit simultaneously. As shown in Fig. 6, that the uncoded Mary frequency shift keying (MFSK) gives a higher transmission rate than an uncoded $\mathrm{BPSK}$ for a fixed $\mathrm{BER}=10^{-5}$ and $M \geq$ 8. MFSK requires a larger bandwidth than an BPSK and this resource is less important than the transmitter power.

The probability of bit error for the noncoherent processing of $M=2^{k}$ orthogonal signals is calculated using the following expression (Proakis 2001, p. 310):

$$
\begin{gathered}
P_{\mathrm{BER}, \mathrm{FSK} \_\mathrm{NC}}=\frac{2^{k-1}}{2^{k}-1} \sum_{n-1}^{M-1}(-1)^{n+1}\left(\begin{array}{c}
M-1 \\
n
\end{array}\right) \times \\
\frac{1}{n+1} \exp \left[-n S N R_{\text {Symbol,FSK }} /(n+1)\right] .
\end{gathered}
$$

The bit error probability as a function of the transmission rate for uncoded coherent MFSK for different M is shown in Fig.
7 whereas the bit error probability as a function of the transmission rate for uncoded noncoherent MFSK for different M is shown in Fig. 8.

Application of channel coding (especially with turbo codes) makes this advantage of MFSK less obvious. It must be also mentioned that the wide spectrum of MFSK is useful for VLBI where the probe's signal may be used for radioastrometric measurements.

\section{Coding gain}

A considerable gain in BER performance can be achieved us $\uparrow$ ing special coding of the bit stream before modulator. The Consultative Commmittee for Space Data System (CCSDS) has recommended the error control standards which were suc cessfully applied in many space missions. Serial concatenation of Read-Solomon outer code $(255,223)$ and convolutional coding (inner code) was used by both NASA and ESA (missions Voyager, Giotto, Galileo). The coding gain obtained with this coding is $\approx 7 \mathrm{~dB}$. This means that $B E R=10^{-5}$ is achieved with $S N R_{b i t}=2.6 d B$ and BPSK (9.6 $d B$ for uncoded BPSK).

Today, the largest part of the promised coding gain can be reached with turbo codes: a combination of parallel concatenation of binary convolutional coders with interleaving and iterative decoding (Divsalar \& Pollara 1995, Dolinar et. al 1998, Habinc et al. 1998, Heegard \& Wicker 1999). Turbo coding provides bit error performance within a few tenths of a $\mathrm{dB}$ of the Shannon limit. The documents recently published by CCSDS show that future missions will use turbo coding (Habinc 1998).

An analytical expression of BER for a particular scheme of turbo code is not known. Many numeric simulations have been made to obtain such estimates. Fig. 9 shows bit-error probabilities for turbo code $($ rate $=1 / 2$ ) and multiple iterations (Sklar 2001). The Shannon limit is approached but the normalized capacity of the channel tends to zero (as in the case of the Shannon's limit).

The following threshold value of turbo code for $S N R_{b i t}$ will be used further: the bit-error probability $P_{B}<10^{-5}$ at $S N R_{b i t}=$ $0.7 \mathrm{~dB}$ (for $1 / 2$ code rate). For binary modulation and $P_{B}<$ $10^{-5}$ a pragmatic Shannon limit $S N R_{\text {bit }}=0.2 \mathrm{~dB}$ (instead of $-1.6 \mathrm{~dB}$ ) is often used for a rate $1 / 2$ code. Thus the errorperformance of turbo code is within $0.5 \mathrm{~dB}$ of the pragmatic Shannon limit (Sklar 2001).

Fig. 10 demonstrates four curves: the dependance of transmission rate for $B E R=10^{-5}$ as a function of distance $R$ in AU for the SKA core, S-band. The curves correspond to uncoded BPSK (threshold $S N R_{b i t, t h r}=9.59 \mathrm{~dB}$ ), turbo code (threshold $S N R_{b i t, t h r}=0.7 \mathrm{~dB}$ ), pragmatic Shannon limit (threshold $S N R_{b i t, t h r}=0.2 \mathrm{~dB}$ ) and theoretical Shannon limit (threshold $\left.S N R_{b i t, t h r}=-1.6 \mathrm{~dB}\right)$.

Table 3 contains the values of transmission rate (bit/sec) in S-band for turbo-coded BPSK, BER $=10^{-5}$, and the SKA core (aperture array), $A_{e f f}=10^{5} \cdot \cos (\alpha) \mathrm{m}^{2}, \alpha$ - zenith angle, $T_{\text {sys }}=40 \mathrm{~K}$, distance $=5 A U$. The transmitter power $P_{t r}$, antenna gain $G_{t r}$ and $\alpha$ are the parameters. 
Table 3: The DtE transmission rate (bit/sec) in S-band for turbo-coded BPSK, $B E R=10^{-5}$ and the SKA core (aperture array), $A_{\text {eff }}=$ $10^{5} \cdot \cos (\alpha) \mathrm{m}^{2}, \alpha$ - zenith angle, $\eta=0.8, T_{\text {sys }}=40 \mathrm{~K}$, distance $=5 \mathrm{AU}$, transmitter power $P_{t r} \cdot 0.75$ and antenna gain $G_{t r}$.

\begin{tabular}{|c|c|c|c|c|}
\hline \multicolumn{5}{|c|}{$\alpha=0^{\circ}$} \\
\hline$P_{t r}, \mathrm{~W}$ & 1.0 & 3.0 & 5.0 & 10.0 \\
\hline$G_{t r}=0 d B$ & 19.74 & 59.21 & 98.69 & 197.38 \\
\hline$G_{t r}=5 d B$ & 62.417 & 187.251 & 312.09 & 624.17 \\
\hline$G_{t r}=10 \mathrm{~dB}$ & 197.38 & 592.14 & 986.90 & 1974.00 \\
\hline \multicolumn{5}{|c|}{$\alpha=30^{\circ}$} \\
\hline$P_{t r}, \mathrm{~W}$ & 1.0 & 3.0 & 5.0 & 10.0 \\
\hline$G_{t r}=0 d B$ & 17.09 & 51.28 & 85.47 & 170.94 \\
\hline$G_{t r}=5 d B$ & 54.06 & 162.16 & 270.27 & 540.55 \\
\hline$G_{t r}=10 \mathrm{~dB}$ & 170.94 & 512.81 & 854.68 & 1709.00 \\
\hline \multicolumn{5}{|c|}{$\alpha=60^{\circ}$} \\
\hline$P_{t r}, \mathrm{~W}$ & 1.0 & 3.0 & 5.0 & 10.0 \\
\hline$G_{t r}=0 d B$ & 9.87 & 29.61 & 49.35 & 98.69 \\
\hline$G_{t r}=5 d B$ & 31.21 & 93.63 & 156.04 & 312.09 \\
\hline$G_{t r}=10 \mathrm{~dB}$ & 98.69 & 296.07 & 493.45 & 986.90 \\
\hline
\end{tabular}

\section{Conclusions}

The analysis described in this memo indicates a significant potential of the SKA as a DtE facility. As the benchmark value, we note that at the communication frequency of 2.3 $\mathrm{GHz}$ (considered to be close to the band of maximum sensitivity of SKA), the turbo-coded DtE link with the SKA core $\left(1.5 \cdot 10^{5} \mathrm{~m}^{2}\right)$ can provide for the transmission rate of $\sim 100$ bps from a $\mathrm{S} / \mathrm{C}$ at the distance of $5 \mathrm{AU}$, transmitter power equal $5 \mathrm{~W}$ and antenna gain $\mathrm{Gt}=0 \mathrm{~dB}$.

In addition to the high sensitivity, illustrated by our calculations, the SKA offers instantaneous frequency coverage from $\sim 100 \mathrm{MHz}$ to $\sim 10 \mathrm{GHz}$, unlike traditional DSN-style facilities able to operate within relatively narrow dedicated frequency bands. Such the frequency agility might be an important asset for supporting communication from planetary probes as a mission back-up, e.g. similar to "eavesdropping" on the Huygens up-link communication to the Cassini spacecraft at the non-standard down-link at the frequency of $2040 \mathrm{MHz}$ (Lebreton et al. 2005). Planetary probes for in situ studies of atmospheres of giant planets (e.g. Jupiter, Saturn) require communication at frequencies below $\sim 1 \mathrm{GHz}$ (see e.g. Marty et al. 2008). In this non-standard communication regime, the SKA also might become an indispensable asset.

We note that MFSK is also convenient for VLBI tracking since it is better suited for group delay measurements than a mono-chromatic carrier.

Our main conclusion is as follows:

The SKA core will be able to receive digital information from a planetary probe or interplanetary $\mathrm{S} / \mathrm{C}$ with a transmitter power of $5 \mathrm{~W}$ from a distance of up to $10 \mathrm{AU}$ with the transmission rate of several tens of bps using BFSK and turbo-coding gain, see Table 4.
Table 4: The DtE transmission rate (bit/sec) at S-band for the SKA core (aperture array), $A_{\text {eff }}=1.5 \cdot 10^{5} \mathrm{~m}^{2}$, zenith angle $\alpha=0, \eta=$ $0.8, T_{\text {sys }}=40 \mathrm{~K}$, transmitter power $P_{t r}=1.0 \cdot 0.75 \mathrm{~W}$ and antenna gain $G_{t r}=0 \mathrm{~dB}$, turbo-coded BPSK and $B E R=10^{-5} ; \mathrm{X}$-band (parabolic dishes), $\eta=0.6, T_{s y s}=60 \mathrm{~K}$; UHF-band (aperture array), $T_{s y s}=70 \mathrm{~K}$, additional 3-dB loss in Earth ionosphere, interplanetary medium and Jupiter atmosphere are taken into account.

\begin{tabular}{c|cccc}
\multicolumn{5}{c}{ UHF-band } \\
\hline \hline$P_{t r}, \mathrm{~W}$ & 1.0 & 3.0 & 5.0 & 10.0 \\
\hline$R=5.0 \mathrm{AU}$ & 5.64 & 16.92 & 28.20 & 56.40 \\
$R=7.0 \mathrm{AU}$ & 2.88 & 8.63 & 14.39 & 28.77 \\
$R=10 \mathrm{AU}$ & 1.41 & 4.23 & 7.05 & 14.1 \\
\hline \multicolumn{5}{c}{ S-band } \\
\hline \hline$P_{t r}, \mathrm{~W}$ & 1.0 & 3.0 & 5.0 & 10.0 \\
\hline$R=5.0 \mathrm{AU}$ & 19.74 & 59.21 & 98.69 & 197.38 \\
$R=7.0 \mathrm{AU}$ & 10.07 & 30.21 & 50.35 & 100.70 \\
$R=10 \mathrm{AU}$ & 4.93 & 14.80 & 24.67 & 49.345 \\
\hline \multicolumn{5}{|c}{$\mathrm{X}$-band } \\
\hline \hline$P_{t r}, \mathrm{~W}$ & 1.0 & 3.0 & 5.0 \\
\hline$R=5.0 \mathrm{AU}$ & 9.869 & 29.61 & 49.35 & 10.0 \\
$R=7.0 \mathrm{AU}$ & 5.035 & 15.11 & 25.18 & 50.69 \\
$R=10 \mathrm{AU}$ & 2.47 & 7.40 & 12.34 & 24.68 \\
\hline \multicolumn{5}{c}{}
\end{tabular}

\section{References}

Blanc M., Alibert Y., Atreya S.K. et al., 2009, Laplace: a mission to Europa and the Jupiter System for ESA's Cosmic Vision Programme, Exp. Astronomy, 23, 849

Chassefière E., Korablev O., Imamura T., et al. 2009, European Venus Explorer (EVE): an in-situ mission to Venus, Exp. Astronomy, 23, 741

Coustenis A., Atreya S.K., Balint T. et al., 2009, TandEM: Titan and Enceladus mission, Exp. Astronomy, 23, 893

Divsalar S. and Pollara F. 1995, Turbo Codes for DeepSpace Communications, TDA Progress Report 42-120, Jet Propulsion Laboratory, Pasadena, California, Feb. 15, 1995 , pp. 29-39.

Dolinar S., Divsalar D. and Pollara F. 1998 Turbo Codes and Space Communications, http://track.sfo.jaxa. jp/spaceops98/paper98/track5/5e011.pdf

Habinc S. et al. 1998, Development Plan for Turbo Encoder Core and Devices Implementing the Updated CCSDS Telemetry Channel Coding Standard, ftp://ftp.estec.esa.nl/pub/vhdl/doc/Turbo.pdf

Heegard, C. and Wicker, S. B., 1999, Turbo coding, Kluwer Academic Publishers, Boston/Dordrecht/London

Jones D. 2004, Spacecraft tracking with the SKA, in Science with the Square Kilometre Array, eds. C. Carilli and S. Rawlings, Elsevier, p. 1537

Lebreton J.-P. et al., 2005, An overview of the descent and landing of the Huygens probe on Titan, Nature 438, 758

Marty B., Guillot T., Coustenis A. et al., 2009, Kronos: exploring the depths of Saturn with probes and remote sensing through an international mission, Exp. Astronomy, 23, 947

Proakis, J. G, 2001, Digital communications. 4th ed. Boston McGraw-Hill 


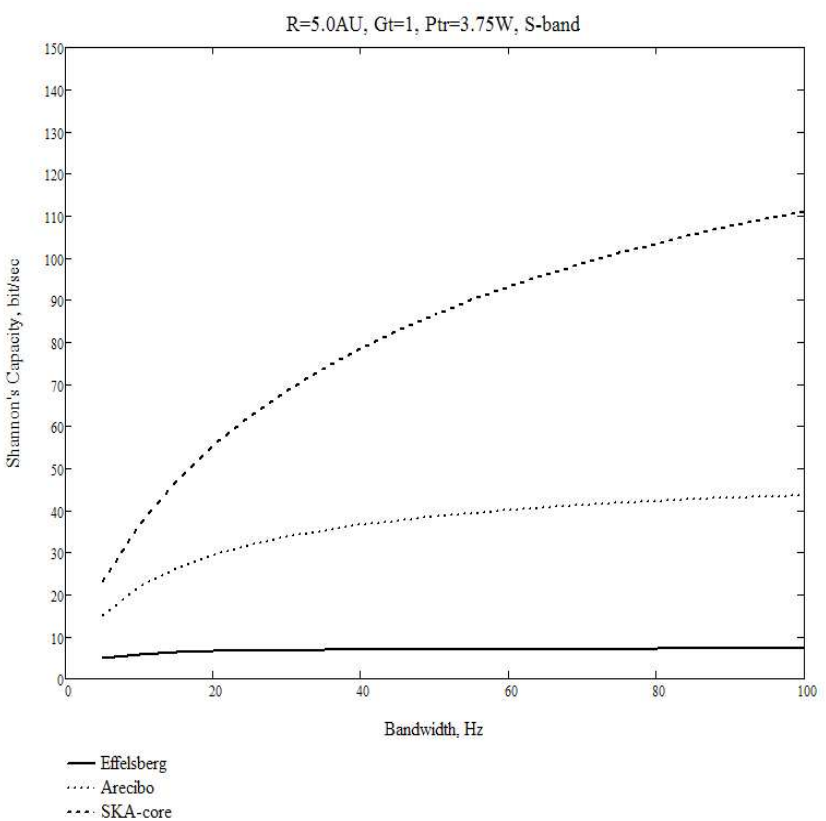

Fig. 1: The channel capacity as a function of bandwidth for several radio telescopes from Table 1 .

Schilizzi R.T. et al., 2007, Preliminary Specifications for the Square Kilometre Array, http://www. skatelescope. org/PDF/Preliminary_SKA_Specifications.pdf

Sklar, B., 2001, Digital communications, Prentice-Hall, Inc., NJ, p.497

Yuen, J. H. et al., 1990, Modulation and Coding for Satellite and Space Communications, Proc. IEEE, vol. 78, n. 7., July 1990, p. 1250

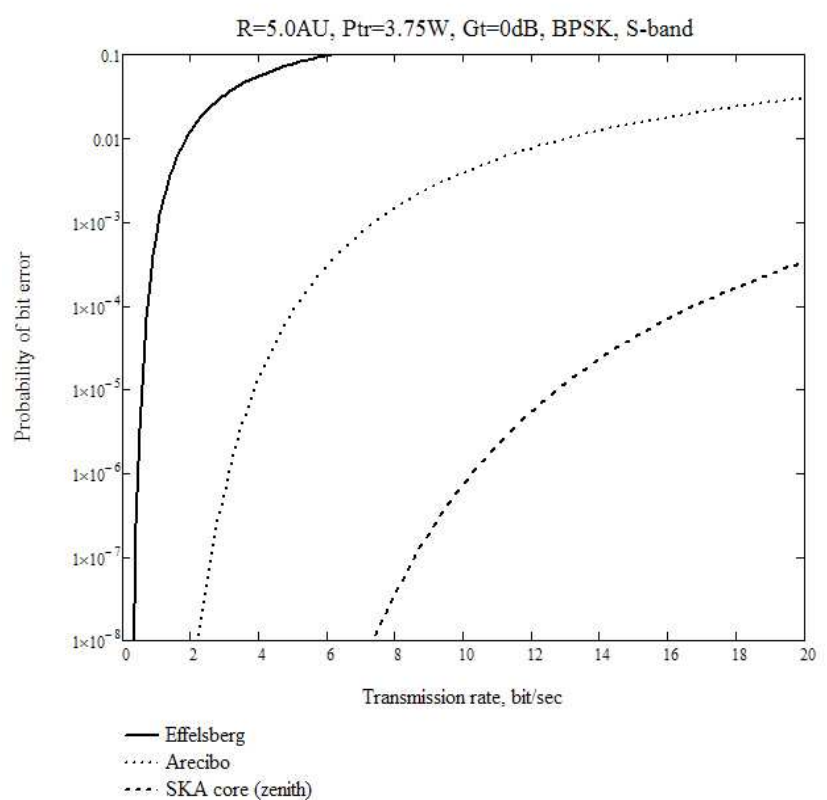

Fig. 2: The bit error probability as a function of the transmission rate for radio telescopes in Table 1, BPSK, $R=5.0 \mathrm{AU}, P_{t r}=3.75 \mathrm{~W}, G_{t r}=$ 1.

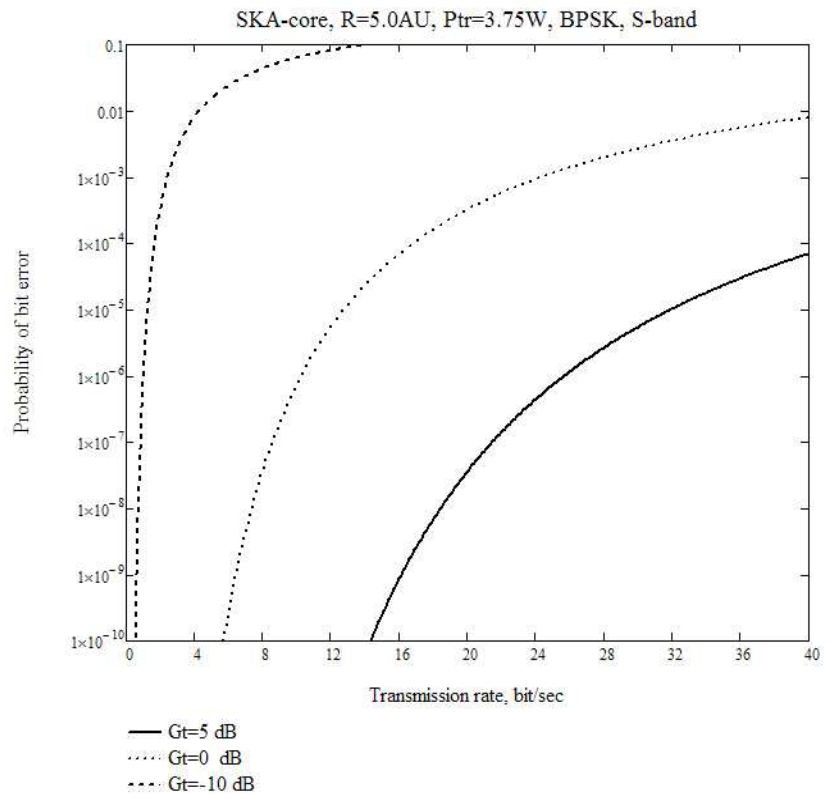

Fig. 3: The bit error probability as a function of the transmission rate for the SKA-core, BPSK, $R=5.0 A U, P_{t r}=3.75 \mathrm{~W}$, transmitter antenna gain is the parameter. 


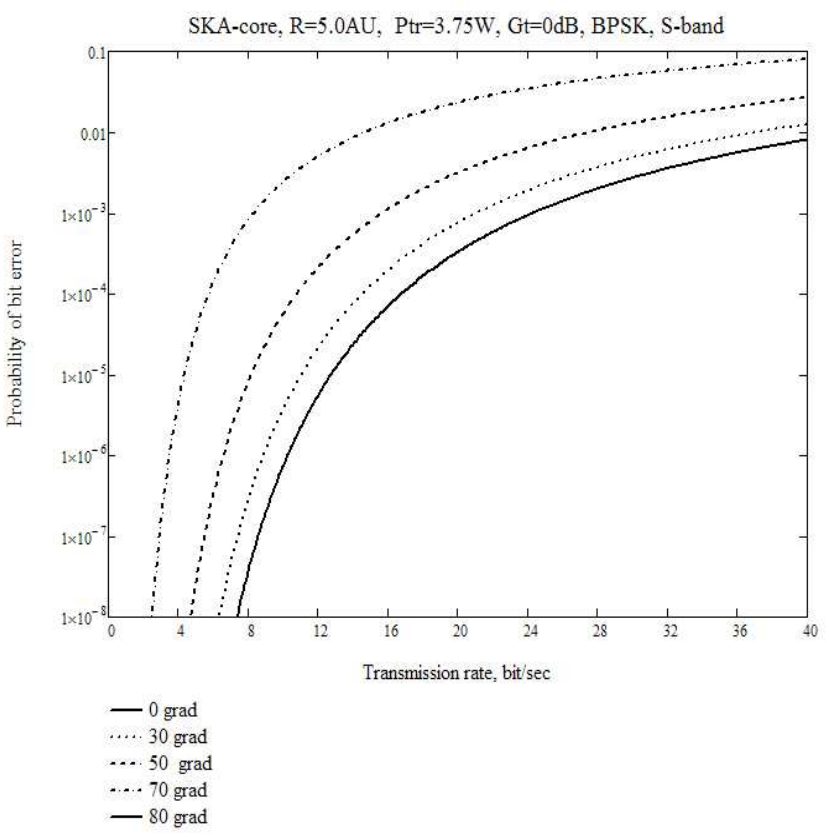

Fig. 4: The bit error probability as a function of the transmission rate for the SKA-core, BPSK, $R=5.0 A U, P_{t r}=3.75 \mathrm{~W}$, zenith angle is the parameter.

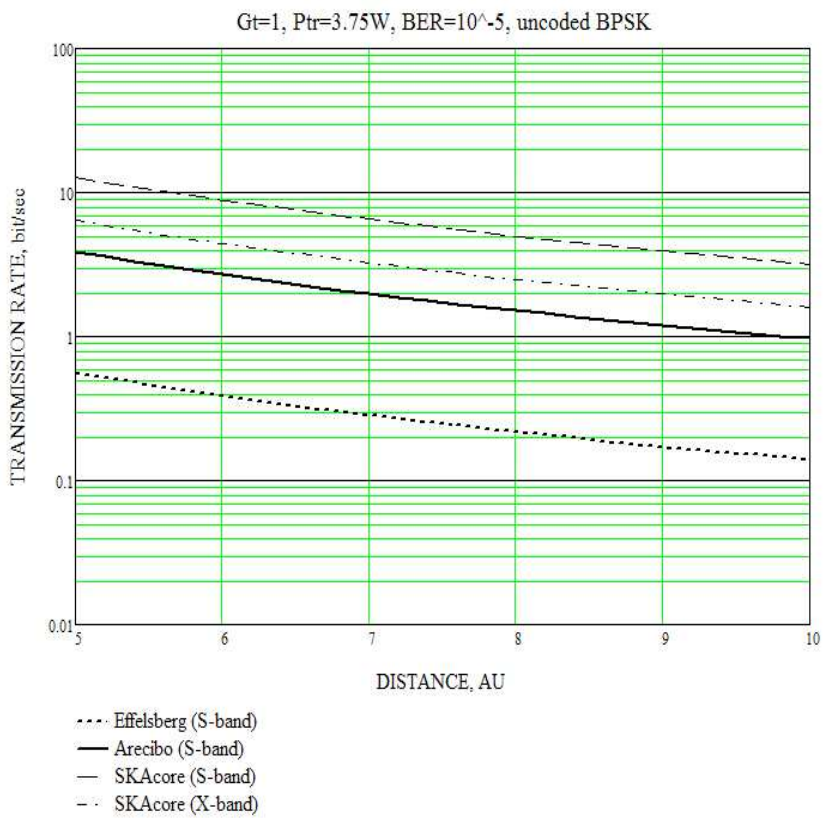

Fig. 5: The transmission rate as a function of distance in AU for radio telescopes in Table 1 and constant $B E R=10^{-5}$, for $P_{t r}=$ $3.75 W, G_{t r}=1$.

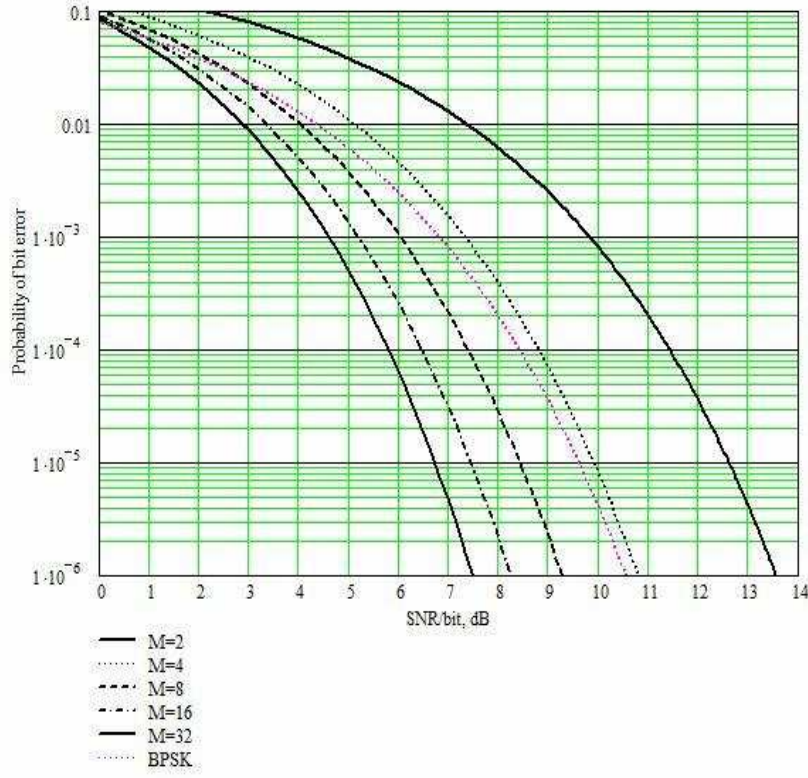

Fig. 6: The bit error probability as a function of $S N R_{b i t}$ for uncoded MFSK (multiple FSK) with the symbol set size is the parameter; BPSK curve is also plotted for comparison.

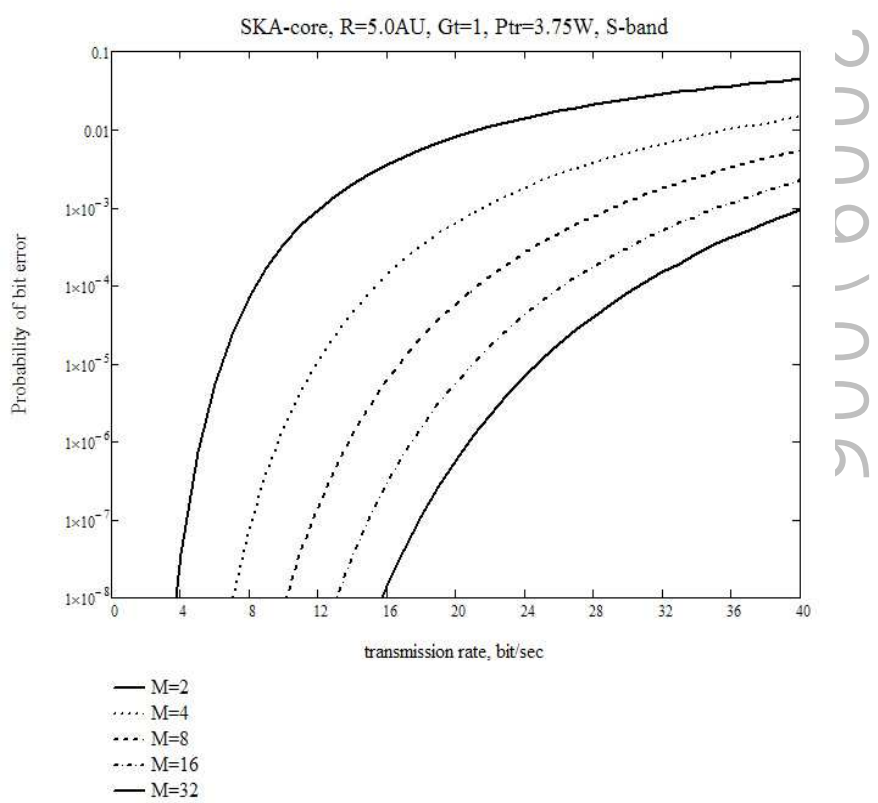

Fig. 7: The bit error probability as a function of the transmission rate for uncoded coherent MFSK with the symbol set size as the parameter. 


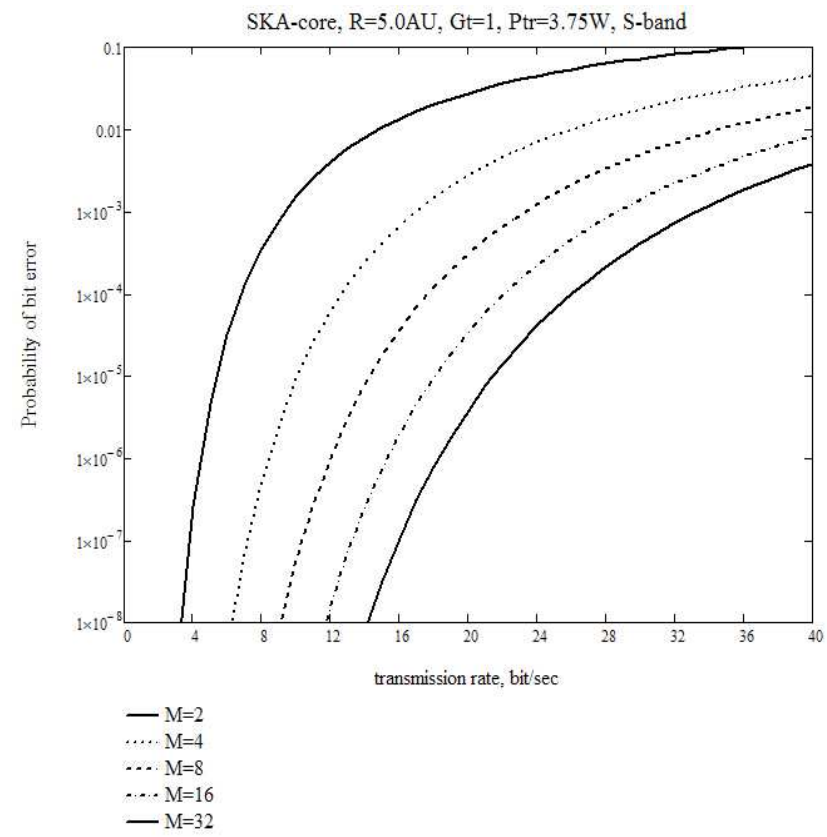

Fig. 8: The bit error probability as a function of the transmission rate for uncoded noncoherent MFSK with the symbol set size as the parameter.

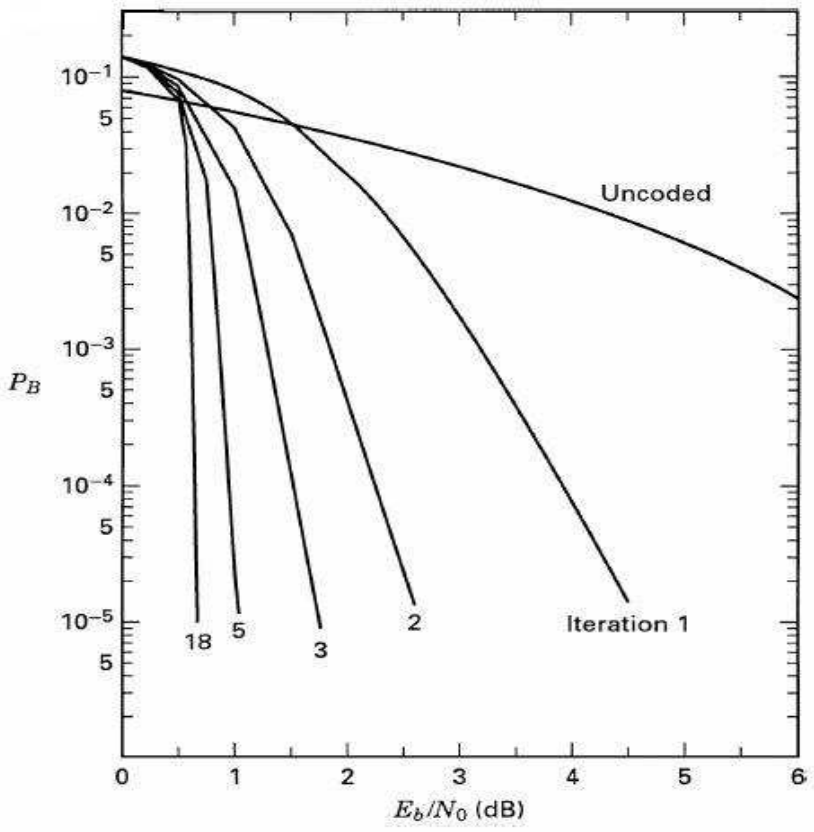

Fig. 9: The bit-error-performance as a function of $S N R_{b i t}$ for turbo code (rate 1/2) and multiple iterations. The Shannon limit of $-1.6 \mathrm{~dB}$ is approached.

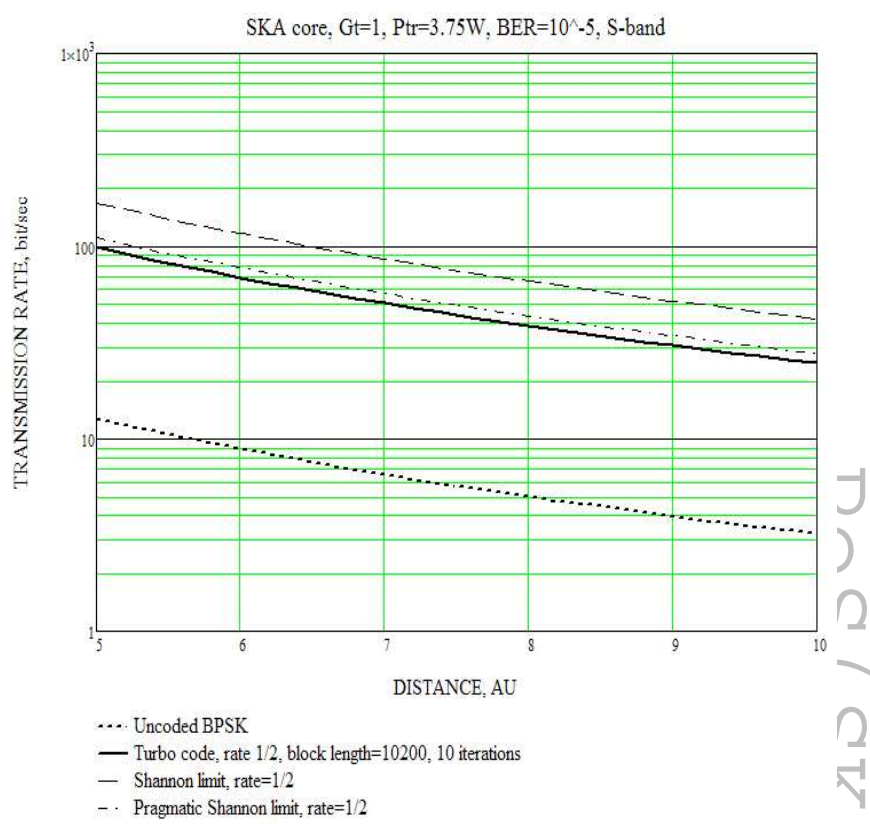

Fig. 10: The transmission rate as a function of distance for uncoded BPSK, turbo code, pragmatic and theoretical Shannon limits: SKA core, $P_{t r}=3.75 \mathrm{~W}, G_{t r}=1, B E R=10^{-5}$. 